\title{
Association between dietary inflammatory index and periodontitis: a cross-sectional and mediation analysis
}

\author{
Vanessa Machado ${ }^{1,2}$, João Botelho ${ }^{1,2, *}$, João Viana ${ }^{1}$, Paula Pereira ${ }^{3}$, Luísa Lopes ${ }^{1}$, Luís Proença \\ 2,4, Ana Sintra Delgado ${ }^{1}$, José João Mendes ${ }^{1,2}$ \\ 1 Clinical Research Unit (CRU), Centro de Investigação Interdisciplinar Egas Moniz (CiiEM), Egas Moniz - \\ Cooperativa de Ensino Superior, Almada, Portugal; vmachado@egasmoniz.edu.pt (V.M.); \\ jpm.viana.1@gmail.com (J.V.); luisabpmlopes@gmail.com (L.L.); anasintradelgado@egasmoniz.edu.pt \\ (A.S.D.) jmendes@egasmoniz.edu.pt (J.J.M.). \\ 2 Evidenced-Based Hub, CiiEM, Egas Moniz - Cooperativa de Ensino Superior, Almada, Portugal; \\ lproenca@egasmoniz.edu.pt (L.P.). \\ 3 Grupo de Estudos em Nutrição Aplicada (GENA), CiiEM, Egas Moniz - Cooperativa de Ensino Superior, \\ Almada, Portugal; pereira.paula1@gmail.com (P.P.). \\ 4 Quantitative Methods for Health Research (MQIS), CiiEM, Egas Moniz - Cooperativa de Ensino Superior, \\ Almada, Portugal \\ * Correspondence: jbotelho@egasmoniz.edu.pt; Tel.: +351-969-848-394 (J.B.)
}

\begin{abstract}
Background: We aimed to assess the association between DII and PD and the mediation effect of DII in the association of PD with systemic inflammation. Using the National Health and Nutrition Examination Survey (NHANES) 2009-2010, 2011-2012 and 2013-2014, participants that received periodontal exam and provided dietary recall data were included. The inflammatory potential of diet was calculated via DII. Periodontitis was defined according to the 2012 case definition. The clinical outcomes of interest were mean periodontal probing (PPD), mean clinical attachment loss (CAL) and thresholds of PPD and CAL. White blood cells (WBC), segmented neutrophils and C-reactive protein (CRP) were used as proxies for systemic inflammation. The periodontal measures were regressed across DII values using adjusted multivariate linear regression. Adjusted mediation analysis appraised the influence of DII in the association of periodontitis and systemic inflammation. 10,178 participants were included. DII was significantly correlated with mean PPD, mean CAL, thresholds of PPD and CAL, WBC, segmented neutrophils and DII ( $\mathrm{p}<0.01)$. A linear regression logistic adjusted for multiple confounding variables confirmed the association between DII and mean PPD ( $B=0.02$, Standard Error [SE]: 0.02, $p<0.001)$ and CAL $(B=-0.02$, SE: 0.01, $\mathrm{p}<0.001)$. The association of mean PPD and mean CAL with both white blood cells and segmented neutrophils were mediated by DII (from 2.1 to $3.5 \%$, $\mathrm{p}<0.001$ ). In the 2009-2010 subset, the association of mean CAL with serum CRP was mediated by DII $(52.0 \%, \mathrm{p}<0.01)$. In conclusion, inflammatory diet and PD may be associated. Also, the inflammatory diet significantly mediated the association of leukocyte counts and systemic inflammation with periodontitis.
\end{abstract}

Keywords: NHANES; periodontal diseases; periodontitis; tooth loss; inflammation; diet; nutrition; oral health

\section{Introduction}

A healthier life is heavily dependent on diet [1-4].The inflammatory burden of diet has brought up great interest considering the anti- and proinflammatory modulating potential of nutrients. Proinflammatory dietary patterns have been associated with some systemic conditions, including cardiovascular diseases [5,6], higher mortality rate in breast cancer survivors [7], rheumatoid arthritis [8] or periodontal disease [9]. Further, the systemic balance of chronic inflammation is more dependent on diet than medication usage, given the frequency of food ingestion [10]. Thus, if the 
status quo of inflammation is controlled, several reactions or diseases determined by inflammatory pathways may be avoided or interrupted. To a certain extent, the inflammatory load of diet fosters a more comprehensive outlook rather than single-nutrient based assessments and with considerable research interest [11]. For this reason, the Dietary Inflammatory Index (DII) was proposed as a literature-based tool to categorize individuals' diets based on global standard values [12,13]. Ever since, DII has been associated with the variation of inflammatory markers [14-16] and implicated in systemic diseases $[17,18]$ and tooth loss [19], but there is weak evidence in periodontitis (PD).

PD is a pandemic condition characterized by a serious inflammatory bone loss, soft tissues damage, gingivorrhagia and malodor [20,21]. This non-communicable disease biofilm-induced, causing an exacerbated reaction from the immune system that destroys the periodontium [22]. PD starts with gingivitis (gum inflammation without bone loss) however, if untreated, can precipitate bone loss and, ultimately, tooth loss [23]. Besides, PD is responsible for a reversible impairment of patient's quality of life $[24,25]$. Some studies demonstrate a link between periodontal disease and nutrition [26-28]. In particular, an anti-inflammatory diet demonstrated therapeutical potential in gingivitis [9], still, regular flavonoid intake, recognized anti-inflammatory compounds, had no association with the risk of PD [29].

The present study explored whether the inflammatory degree of diet, measured via DII, is associated with PD. Secondly, we investigated the mediation effect of DII in the association of PD with systemic inflammation (assessed through white blood cells count [WBC], segmented neutrophils and C-reactive protein [CRP]). We hypothesized that worse periodontal clinical measures are associated with the inflammatory load originated in the diet, and that this load might significantly mediate the systemic inflammatory burden caused by PD.

\section{Materials and Methods}

\subsection{Study Design and Participants}

This secondary analysis used data from the NHANES (National Health and Nutrition Examination Survey) marked between 2009-2010, 2011-2012 and 2013-2014. The NHANES is a stratified multistage research program conducted by the National Center for Health Statistics (NCHS) of the civilian non-institutionalized population in the 50 stages of the United States (US) and the District of Columbia. NHANES aims to assess the health and nutritional status and to monitor changes over time. The way of collecting data is through interviews, physical and laboratory exams, and all NHANES 2009-2014 were reviewed and approved by the Centers for Disease Control (CDC) and Prevention National Center for Health Statistics Research (NCHS) Ethics Review Board, and all included participants provided written informed consent.

For the purpose of this study, NHANES 2009-2010; 2011-2012 and 2013-2014 data were retrieved with the following inclusion criteria: participants aged 18 or over, who received periodontal examination and answered the dietary interview for the total nutrient intakes. We followed the extension of the STrengthening the Reporting of OBservational studies in Epidemiology for Nutritional Epidemiology (STROBE-Nut) [30].

\subsection{Diet and Dietary Inflammatory Index}

The DII is a literature-based tool designed to categorize individuals' diets on a continuum from maximally anti-inflammatory to maximally pro-inflammatory [12,13]. Briefly, the DII is a scoring algorithm based on dietary intake data that are linked to a regionally representative world database to estimate a mean and standard deviation (SD) for each parameter. In this study, from a possible total of 45 food parameters, we calculated the DII score for 26 food parameters available. Eugenol, garlic, ginger, niacin, onion, saffron, saturated fat, trans fat, turmeric, green/black tea, flavan-3-ol, flavones, flavonols, flavonones, anthocyanidins, isoflavones, pepper, thyme/oregano, rosemary were not included because no information was available. First, we calculated the z-score of each food 
parameter and for each participant. Second, each individual z-score was converted to a centered percentile. Third, each centered percentile was multiplied by the standardized overall inflammatory effect score [12]. Then, the DII score was summed for each participant. The final score is a continuous measure, interpreted as strongly anti-inflammatory (the lowest score) to strongly proinflammatory (the highest score), respectively [12].

\subsection{Periodontal examination}

Full-month periodontal examination at six sites per tooth (mesiobuccal, buccal, distobuccal, mesiolingual, lingual, and distolingual) was conducted by trained calibrated examiners as described elsewhere [31]. Probing pocket depth (PPD) and gingival recession measures were made using a HUFriedy periodontal probe graduated in 2-mm increments. Clinical attachment loss (CAL) was calculated as the difference between PPD and gingival recession. The PD diagnosis and staging were carried out following the CDC and Prevention-American Academy of Periodontology consensus for epidemiologic studies recommendation [32]. In this sense, mild PD was defined as $\geq 2$ interproximal sites with CAL $\geq 3 \mathrm{~mm}$ and $\geq 2$ interproximal sites with PPD $\geq 4 \mathrm{~mm}$ (not on the same tooth) or 1 site with PPD $\geq 5 \mathrm{~mm}$. Moderate PD was defined as $\geq 2$ interproximal sites with CAL $\geq 4 \mathrm{~mm}$ (not on the same tooth) or $\geq 2$ interproximal sites with PPD $\geq 5 \mathrm{~mm}$, also not on the same tooth. Severe PD was defined as the presence of $\geq 2$ interproximal sites with CAL $\geq 6 \mathrm{~mm}$ (not on the same tooth) and $\geq 1$ interproximal site with PPD $\geq 5 \mathrm{~mm}$. Additionally, periodontal epithelial surface area (PESA) for each tooth and for all participants were calculated using a Microsoft Excel spreadsheet [33].

\subsection{Covariates}

Self-reported socio-demographic characteristics regarding age, gender, race or ethnicity (i.e., Mexican American, Non-Hispanics White, Non-Hispanics Black, other Hispanics and other races) and higher education level (i.e., less than high school, complete high school or similar, higher than high school) was collected for each wave. Smoking status was subdivided as never (smoked less than 100 cigarettes in life and not currently smoking), former (smoked at least 100 cigarettes in life and not currently smoking), active smoker (smoked at least 100 cigarettes in life and currently smoking). Body mass index (BMI) was calculated as weight in kilograms divided by height in meters squared. Systolic and diastolic blood pressure (SBP and DBP) measurements were determined by trained and calibrated examiners. Blood levels data included WBC Count $\left(10^{9} / \mathrm{L}\right)$, segmented neutrophils $\left(10^{9} / \mathrm{L}\right)$, vitamin D (mg/dL), hemoglobin A1c (Hba1c) (\%), total cholesterol and CRP (mg/dL).

\subsection{Data management, test methods and analysis}

We collected data from the NHANES 2009-2010; 2011-2012 and 2013-2014 were uploaded through SAS Universal Viewer for Windows and handled with IBM SPSS Statistics version 25.0 for Macintosh (Armonk, New York, IBM Corp.) To make the periodontal diagnosis, we export our data to an Microsoft Excel spreadsheet with the respective algorithms to be able to calculate PESA (as in [34]). Descriptive measures are reported through mean (SD) for continuous variables, and a number of cases (n) and percentage (\%) for categorical variables.

The correlation between the DII and CRP with the periodontal clinical measures, WBC and segmented neutrophils was assessed by the Pearson correlation coefficient. Further, a multivariate stepwise adjusted linear regression procedure was used to model the influence of DII and CRP on the periodontal clinical measures, WBC and segmented neutrophils. Model variables were selected among clinical and demographic characteristics. Following the initial crude model (model 1), seven progressively adjusted models were generated (model 2 - includes adjustment for DII and age; model 3 - includes adjustment for DII, age and BMI; model 4 - includes adjustment for DII, age, BMI and number of chronic medical conditions; model 5 - includes adjustment for DII, age, BMI, number of chronic medical conditions and SBP; model 6- includes adjustment for DII, age, BMI, number of 
chronic medical conditions, SBP and Hba1c; model 7-includes adjustment for DII, age, BMI, number of chronic medical conditions, SBP, Hba1c and total cholesterol; model 8-includes adjustment for DII, age, BMI, number of chronic medical conditions, SBP, Hba1c, total cholesterol and vitamin D levels).

To investigate the potential mediating effect of DII on the association between mean CAL/PPD (exposure) and CRP/WBC (outcome) we used multivariable regression analysis. Three pathways (a, $\mathrm{b}$ and $\mathrm{c}$ ) were used to assess the mediation. Total effect evaluated the relationship between mean CAL/PPD (exposure) and CRP/WBC (outcome). Path a assessed the relationship between mean $\mathrm{CAL} / \mathrm{PPD}$ and DII (mediator). Path $\mathrm{b}$ measured the relationship between DII (mediator) and CRP/WBC (outcome). The influence of DII on the link between mean CAL/PPD and CRP/WBC were assessed through path c (direct effect). The proportion of the mediated effect was calculated using the following formula: (total effect - direct effect) x 100 / Btotal effect. All models were conducted adjusted for sociodemographic variables (age, gender, race, education level), health behaviors (minutes of sedentarism, smoking habit, alcohol use), BMI, number of missing teeth and systemic status (number of medical conditions, SBP, DBP, Hba1c, total cholesterol and vitamin D). A value of $\mathrm{p}<0.05$ was considered significant.

\section{Results}

\subsection{Characteristics of the Study Sample}

From an initial sample of 30,468 participants, 20,290 were excluded considering the exclusion criteria, resulting in a final sample of 10,178 patients. The characteristics of this sample are depicted in Table 1. The overall sample had an average age of 52.0 (14.3) years and mostly were female participants (50.7\%). This sample was predominantly composed by Non-Hispanic White participants (30.7\%) with higher education level (55.2\%). Regarding smoking habits, most of the participants never smoked $(56.2 \%)$, and $18.6 \%$ were active smokers. Also, this sample had an average BMI of 29.1 (7.0) Kg/m² and high prevalence of PD (59.4\%). Particularly, CRP values were only available for the sample of 2009-2010 NHANES.

Table 1. Participants characteristics.

\begin{tabular}{|c|c|c|c|c|}
\hline Variable & $\begin{array}{l}\text { NHANES } \\
2009-2010 \\
(n=3,238)\end{array}$ & $\begin{array}{l}\text { NHANES 2011- } \\
2012(n=3,323)\end{array}$ & $\begin{array}{l}\text { NHANES 2013- } \\
2014(n=3,617)\end{array}$ & $\begin{array}{l}\text { NHANES } \\
2009-2014 \\
(n=10,178)\end{array}$ \\
\hline $\begin{array}{l}\text { Age, mean (SD) } \\
\text { (years) }\end{array}$ & $52.2(14.4)$ & $51.8(14.2)$ & $52.0(14.3)$ & $52.0(14.3)$ \\
\hline \multicolumn{5}{|l|}{ Gender, n(\%) } \\
\hline Male & $1623(50.1)$ & $1643(49.4)$ & $1752(48.4)$ & $5018(49.3)$ \\
\hline Female & 1615 (49.9) & $1680(50.6)$ & 1865 (51.6) & $5160(50.7)$ \\
\hline \multicolumn{5}{|l|}{ Race/ethnicity, n (\%) } \\
\hline Mexican American & $585(18.1)$ & $355(10.7)$ & $491(13.6)$ & $1431(14.1)$ \\
\hline Non-Hispanics White & $339(10.5)$ & $1226(36.9)$ & $1562(43.2)$ & $3127(30.7)$ \\
\hline Non-Hispanic Black & $1555(48.0)$ & $839(25.2)$ & $704(19.5)$ & 3098 (30.4) \\
\hline Other Hispanic & $586(18.1)$ & $343(10.3)$ & $322(8.9)$ & $1251(12.3)$ \\
\hline Other race & $173(5.3)$ & $560(16.9)$ & $538(14.9)$ & $1271(12.5)$ \\
\hline Education & & & & \\
\hline$<$ High school & $401(12.4)$ & $318(9.6)$ & $283(7.9)$ & $1000(9.8)$ \\
\hline High school & 1203 (37.2) & 1135 (34.2) & 1217 (33.7) & 3555 (34.9) \\
\hline > High school & $1634(50.5)$ & $1870(56.2)$ & $2117(58.5)$ & $5623(55.2)$ \\
\hline Smoking status, $\mathrm{n}(\%)$ & & & & \\
\hline
\end{tabular}




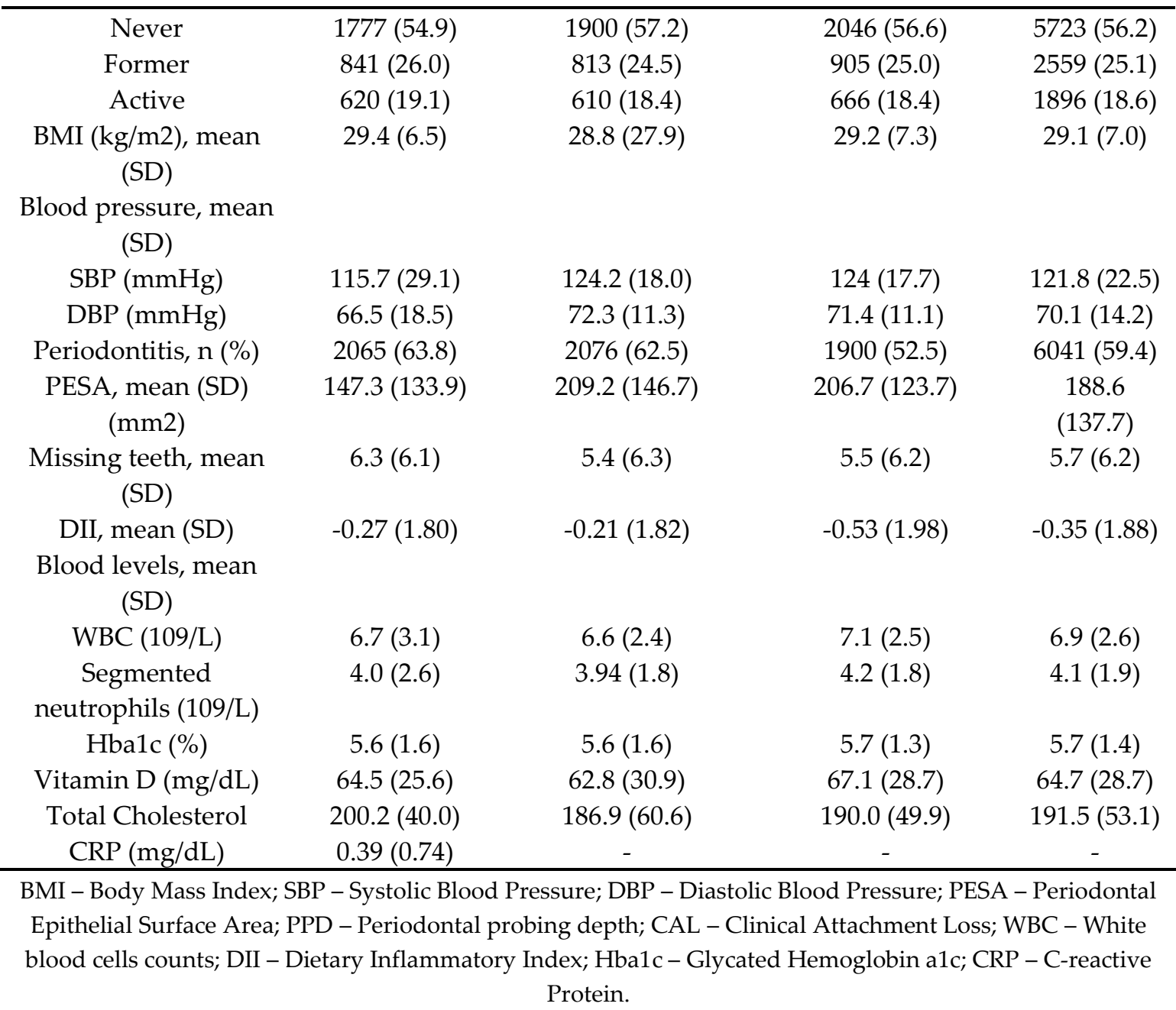

\subsection{DII, PD and systemic inflammation}

We investigated the correlation of DII and CRP with periodontal measures, WBC and segmented neutrophils (Table 2). In the overall sample, DII was significantly correlated with mean PPD, mean CAL, thresholds of PPD and CAL, WBC and segmented neutrophils $(p<0.01)$. The measures of PESA revealed were not significantly correlated with both DII and CRP ( $p>0.05)$. Additionally, CRP had a significant correlation with mean PPD, mean CAL, thresholds of PPD and CAL, WBC, segmented neutrophils and DII $(\mathrm{p}<0.01)$.

Next, we examined the linear association of DII in a crude (model 1) and adjusted models (models 2-8) for mean PPD, mean CAL, $n$ sites PPD $\geq 5$ and CAL $\geq 5$ (Table 3). Linear regression models confirmed that DII is significantly associated with mean PPD ( $ß$ coefficient $=-0.06, \mathrm{SE}=0.01$, $\mathrm{p}<0.001$ ), mean CAL ( $\beta$ coefficient $=-0.08, \mathrm{SE}=0.01, \mathrm{p}<0.001), \mathrm{n}$ sites PPD $\geq 5$ ( $\beta$ coefficient $=-0.01$, $\mathrm{SE}=0.00, \mathrm{p}<0.001)$ and $\mathrm{n}$ sites $\mathrm{CAL} \geq 5(ß$ coefficient $=-0.02, \mathrm{SE}=0.00, \mathrm{p}<0.001)$.

Table 2. Correlation between DII score with periodontal clinical measures, circulating leukocyte levels for the overall sample $(n=10,178)$ and C-Reactive Protein for the NHANES 2009-2010 sample $(\mathrm{n}=3,238)$.

\begin{tabular}{ccc}
\hline & NHANES & NHANES 2009-2010 \\
& $\begin{array}{c}\mathbf{2 0 0 9 - 2 0 1 4} \\
(\mathbf{n}=\mathbf{1 0 , 1 7 8})\end{array}$ & $(\mathbf{n = 3 , 2 3 8 )}$ \\
\hline Variable & DII & CRP \\
\hline PESA $\left(\mathrm{mm}^{2}\right)$ & -0.019 & 0.008 \\
Mean PPD $(\mathrm{mm})$ & $-0.047^{* *}$ & $0.055^{* *}$ \\
\hline
\end{tabular}




\begin{tabular}{ccc}
\hline Mean CAL $(\mathrm{mm})$ & $-0.042^{* *}$ & $0.062^{* *}$ \\
n sites PPD $\geq 5 \mathrm{~mm}(\%)$ & $-0.039^{* *}$ & $0.070^{* *}$ \\
n sites PPD $\geq 7 \mathrm{~mm}(\%)$ & $-0.030^{* *}$ & $0.056^{* *}$ \\
n sites CAL $\geq 5 \mathrm{~mm} \mathrm{( \% )}$ & $-0.037^{* *}$ & $0.046^{* *}$ \\
n sites CAL $\geq 7 \mathrm{~mm}(\%)$ & $-0.030^{* *}$ & $0.068^{* *}$ \\
WBC $\left(10^{9} / \mathrm{L}\right)$ & $-0.046^{* *}$ & $0.221^{* *}$ \\
Segmented neutrophils (109/L) & $-0.039^{* *}$ & $0.232^{* *}$ \\
DII & - & $0.103^{* *}$
\end{tabular}

Pearson correlation, ${ }^{*} \mathrm{p}<0.05,{ }^{* *} \mathrm{p}<0.01$. PESA - Periodontal Epithelial Surface Area; PPD - Periodontal probing depth; CAL - Clinical Attachment Loss; WBC - White blood cells counts; DII - Dietary Inflammatory Index; CRP - C-reactive Protein.

Table 3. Crude and adjusted linear regression models and correspondent standard error towards mean PPD, mean CAL, PPD $\geq 5$ and CAL $\geq 5$ with DII for the overall samples $(n=10,178)$.

\begin{tabular}{ccccc}
\hline $\begin{array}{c}\text { NHANES 2009-2014 } \\
(\mathbf{n}=\mathbf{1 0 , 1 7 8})\end{array}$ & $\begin{array}{c}\text { Mean PPD } \\
\mathbf{( m m})\end{array}$ & $\begin{array}{c}\text { Mean CAL } \\
(\mathbf{m m})\end{array}$ & $\begin{array}{c}\text { n sites PPD } \geq 5 \\
\mathbf{m m}(\mathbf{0})\end{array}$ & $\begin{array}{c}\text { n sites CAL } \geq 5 \\
\mathbf{m m}(\%)\end{array}$ \\
\hline Model 1 & $0.02(0.02)^{* * *}$ & $-0.02(0.01)^{* * *}$ & $0.00(0.00)^{* * *}$ & $0.00(0.00)^{* * *}$ \\
Model 2 & $0.02(0.00)^{* * *}$ & $-0.02(0.01)^{* * *}$ & $0.00(0.00)^{* * *}$ & $0.00(0.00)^{* * *}$ \\
Model 3 & $0.02(0.00)^{* * *}$ & $-0.02(0.01)^{* * *}$ & $0.00(0.00)^{* * *}$ & $0.00(0.00)^{* * *}$ \\
Model 4 & $0.02(0.00)^{* * *}$ & $-0.02(0.01)^{* * *}$ & $0.00(0.00)^{* * *}$ & $0.00(0.00)^{* * *}$ \\
Model 5 & $0.02(0.00)^{* * *}$ & $-0.02(0.01)^{* * *}$ & $0.00(0.00)^{* * *}$ & $0.00(0.00)^{* * *}$ \\
Model 6 & $0.02(0.00)^{* * *}$ & $-0.02(0.01)^{* * *}$ & $0.00(0.00)^{* * *}$ & $0.00(0.00)^{* * *}$ \\
Model 7 & $0.02(0.00)^{* * *}$ & $-0.02(0.01)^{* * *}$ & $0.00(0.00)^{* * *}$ & $0.00(0.00)^{* * *}$ \\
Model 8 & $0.02(0.00)^{* * *}$ & $-0.02(0.01)^{* * *}$ & $0.00(0.00)^{* *}$ & $0.00(0.00)^{* * *}$ \\
\hline
\end{tabular}

Model 1 - Unadjusted model for dietary inflammatory index (DII); Model 2 - Includes adjustment for DII and age; Model 3 - Includes adjustment for DII, age and body mass index (BMI); Model 4 - Includes adjustment for DII, age, BMI and number of chronic medical conditions; Model 5 - Includes adjustment for DII, age, BMI, number of chronic medical conditions and systolic blood pressure (SBP); Model Includes adjustment for DII, age, BMI, number of chronic medical conditions, SBP and hemoglobin A1c (Hba1c); Model 7-Includes adjustment for DII, age, BMI, number of chronic medical conditions, SBP, Hba1c and total cholesterol; Model 8 -Includes adjustment for DII, age, BMI, number of chronic medical conditions, SBP, Hba1c, total cholesterol and vitamin D levels. ${ }^{*} \mathrm{p}<0.05 ;{ }^{* *} \mathrm{p}<0.01 ;{ }^{* *} \mathrm{p}<0.001$. PPD - Periodontal probing depth; CAL - Clinical Attachment Loss.

\subsection{Mediation Analyses}

After confirming the aforementioned multivariate associations, we constructed a mediation model to explore the relationship between one independent variable (WBC, segmented neutrophils, CRP) and two dependent variables (mean PPD and mean CAL) for the overall sample (Table 4), and one independent variable for the NHANES 2009-2010 (CRP) (Table 5). As a mediator, we considered the DII score. All mediation analyses were computed adjusted for sociodemographic variables (age, gender, race, education), health behaviors (minutes of sedentarism, smoking habit, alcohol use), BMI, and systemic status (number of medical conditions, SBP, DBP, Hba1c, total cholesterol, vitamin D). A statistically significant model was observed when DII was included as a mediator of the association between mean PPD and segmented neutrophils. An indirect effect (3.5\%) of mean PPD on segmented neutrophils mediated by DII was confirmed $(\beta=0.00, \mathrm{SE}=0.00)$ (Table 4$)$. Similarly, a statistically model was obtained when DII was considered as a mediator in the association of mean CAL with 
WBC, with a statistically significant indirect effect $2.1 \%$. Further, mean CAL mediated by DII had a significant indirect effect in CRP level (52.0\%) (Table 5).

Table 4. Mediation analysis of the effects of systemic inflammatory elements on the association of periodontal measures (mean PPD and mean CAL - Exposures) with white blood cells and segmented neutrophils $(\mathrm{n}=10,178)$.

\begin{tabular}{|c|c|c|c|c|c|c|}
\hline $\begin{array}{l}95 \% \text { Coefficient } \\
\text { interval }(95 \% \mathrm{CI})\end{array}$ & Path a & Path $b$ & $\begin{array}{c}\text { Path c (Direct } \\
\text { Effect) }\end{array}$ & $\begin{array}{c}\text { Mediated } \\
\text { Effect }\end{array}$ & $\begin{array}{l}\text { Total } \\
\text { Effect }\end{array}$ & $\begin{array}{c}\text { Proportion } \\
\text { Mediated (\%) }\end{array}$ \\
\hline \multicolumn{7}{|c|}{ Exposure = Mean PPD } \\
\hline \multirow[t]{3}{*}{ WBC $\left(10^{9} / \mathrm{L}\right)$} & 0.14 & 0.04 & $0.21(0.06)^{* * *}$ & $0.01(0.00)$ & 0.21 & 2.7 \\
\hline & $(0.04)^{*}$ & $(0.02)^{*}$ & & & $(0.06)^{*}$ & \\
\hline & ** & ** & & & ** & \\
\hline Segmented & 0.14 & 0.04 & $0.15(0.04)^{* * *}$ & $0.00(0.00)$ & 0.16 & 3.5 \\
\hline Neutrophils & $(0.04)^{*}$ & $(0.02)^{*}$ & & & $(0.04)^{*}$ & \\
\hline$\left(10^{9} / \mathrm{L}\right)$ & ** & $* *$ & & & ** & \\
\hline \multicolumn{7}{|c|}{ Exposure = Mean CAL } \\
\hline \multirow[t]{3}{*}{ WBC (109/L) } & 0.10 & 0.04 & $0.17(0.04)^{* * *}$ & $0.00(0.00)$ & 0.17 & 2.4 \\
\hline & $(0.03)^{*}$ & $(0.02)^{*}$ & & & $(0.04)^{*}$ & \\
\hline & ** & ** & & & ** & \\
\hline Segmented & 0.10 & 0.03 & $0.13(0.03)^{* * *}$ & $0.00(0.00)$ & 0.14 & 2.1 \\
\hline Neutrophils & $(0.03)^{*}$ & $(0.02)^{*}$ & & & $(0.03)^{*}$ & \\
\hline$(109 / \mathrm{L})$ & $* *$ & & & & $* *$ & \\
\hline
\end{tabular}

All models were conducted adjusted for sociodemographic variables (age, gender, race, education), health behaviors (minutes of sedentarism, smoking habit, alcohol use), body mass index, number of missing teeth and systemic status (number of medical conditions, systolic blood pressure, diastolic blood pressure, hemoglobin A1c, total cholesterol, vitamin D). ${ }^{*} p<0.05 ;{ }^{* *} p<0.01 ;{ }^{* * *} p<0.001$. Abbreviations: CAL - clinical attachment loss; DII - dietary inflammatory index, PPD - Periodontal Pocket Depth; WBC - white blood cells. 95\% CI - 95\% confidence interval. 5000 number of bootstrap samples. WBC - White Blood Cells; PPD - Periodontal probing depth; CAL - Clinical Attachment Loss.

Table 5. Mediation analysis of the effects of systemic inflammatory elements on the association of periodontal measures (mean PPD and mean CAL - Exposures) with C-reactive protein $(n=3,238)$.

\begin{tabular}{|c|c|c|c|c|c|c|}
\hline $\begin{array}{l}95 \% \text { Coefficient } \\
\text { interval }(95 \% \mathrm{CI})\end{array}$ & Path a & Path b & $\begin{array}{l}\text { Path c } \\
\text { (Direct } \\
\text { Effect) }\end{array}$ & $\begin{array}{c}\text { Mediated } \\
\text { Effect }\end{array}$ & $\begin{array}{l}\text { Total } \\
\text { Effect }\end{array}$ & $\begin{array}{c}\text { Proportion } \\
\text { Mediated } \\
(\%)\end{array}$ \\
\hline \multicolumn{7}{|c|}{ Exposure = Mean PPD } \\
\hline CRP (mg/dL) & $\begin{array}{c}0.09 \\
(0.07) \\
\end{array}$ & $\begin{array}{c}0.04 \\
(0.01)^{* *} \\
\end{array}$ & $0.08(0.04)$ & $0.00(0.00)$ & $\begin{array}{c}0.07 \\
(0.04)^{*}\end{array}$ & NA \\
\hline \multicolumn{7}{|c|}{ Exposure = Mean CAL } \\
\hline CRP (mg/dL) & $\begin{array}{c}0.13 \\
(0.05)^{*}\end{array}$ & $\begin{array}{c}0.04 \\
(0.01)^{* *}\end{array}$ & $0.06(0.03)^{*}$ & $0.01(0.00)$ & $\begin{array}{c}0.07 \\
(0.03)^{* *}\end{array}$ & 52.0 \\
\hline
\end{tabular}

All models were conducted adjusted for sociodemographic variables (age, gender, race, education), health behaviors (minutes of sedentarism, smoking habit, alcohol use), body mass index, number of missing teeth and systemic status (number of medical conditions, systolic blood pressure, diastolic blood pressure, hemoglobin 
A1c, total cholesterol, vitamin D). Abbreviations: CAL - Clinical Attachment Loss; DII - Dietary Inflammatory Index, PPD - Periodontal Pocket Depth. 95\% CI - 95\% Confidence Interval. ${ }^{*} p<0.05 ;{ }^{* *} p<0.01 ;{ }^{* *} p<0.001$. 5000 number of bootstrap samples. CRP - C-reactive Protein; PPD - Periodontal probing depth; CAL - Clinical Attachment Loss.

\section{Discussion}

Overall, in this cross-sectional study of American participants in a national representative survey, a pro-inflammatory diet was linked with worse periodontal measures, and therefore an association was found. Also, the dietary inflammatory level consistently mediated the association between periodontal measures and systemic inflammation levels.

Until now, only one study had employed the DII tool to appraise the association of inflammatory diet with oral problems, as an anti-inflammatory diet was associated with less missing teeth [19]. Interestingly, the sample included in Kotsakis et al. [19] (NHANES 2009-2010 and 2011-2012) was also included in our study, though we also added the NHANES wave of 2013-2014. For this reason, the number of missing teeth was accounted as a confounding variable to the adjusted analyses. Hence, this study is the first to employ the DII instrument in such a comprehensive and large group of patients diagnosed with PD.

The scientific quest on how nutrition impacts periodontal health has gained wide interest. Dietary behavior was proposed to affect periodontal inflammation in a clinically relevant fashion [35]. Despite the fact that anti- or proinflammatory diets are still poorly studied in periodontal research, several other diet types that encompass inflammatory components have been studied in the past. For instance, individuals that consume a Mediterranean diet, described as anti-inflammatory, were associated with lower levels of periodontal bacteria [36], yet this dietary pattern had no effect on insulin resistance in PD patients [37]. Furthermore, probiotic-rich diet presented contradictory effects on periodontal disease [38-40] and vegan diet did not demonstrate a direct relationship with PD despite influencing salivary microbiota [41]. Regarding particular foods, high-intake doses of cocoa were associated with reduced levels of oxidative stress in PD cases [42]. However, lack of consistency regarding the inflammatory load of diet may explain previous inconsistent results and support the importance of using a comprehensive tool as DII in epidemiological and clinical settings.

A landmark investigation demonstrated that gum bleeding consistently decreased (from $34.8 \%$ to $12.6 \%$ of the overall mouth) after 4 weeks under a regimen of no oral hygiene and no processed diet intake. The lack of oral hygiene led to significant plaque accumulation, yet the absence of processed diet consumption may have contributed to low gingival inflammation [43], however, this study lacked a control group to confirm the observed association. Later, a 4-week low-carbohydrate diet (rich in Omega-3 fatty acids, vitamin C and D, antioxidants and fibers) reduced gingival and periodontal inflammation, in a randomized controlled trial [44]. In a recent controlled trial, a 4-week anti-inflammatory diet significantly improved gingivitis but had no impact on the microbiome composition and serum levels of inflammation [9]. Nonetheless, the results provided by these trials emphasize the potential of combining appropriate oral health routines with low-inflammatory diets.

From a systemic perspective, a pro-inflammatory diet contributes to higher values of systemic inflammation [45]. In fact, DII is a tool that consistently reflects the levels of six inflammatory markers: interleukin (IL)-1 $\beta$, IL-4, IL-6, IL-10, TNF- $\alpha$ and CRP [12]. The possible biological mechanism that links diet and CRP is hypothesized to be based on the components of the inflammatory diet that indirectly promote the activation of CRP, thus leading to greater production of this protein $[15,16,46]$. In detail, the consumption of the so-called pro-inflammatory elements causes an increase in circulating IL levels (particularly IL-6, IL-1 $\beta$ or IL-8) [47]. In response, CRP is dramatically produced in hepatic cells and released into circulation, fostering an even more inflamed systemic status [47]. 
On the other hand, PD causes a local inflammatory reaction that can evolve systemically, increasing WBC and segmented neutrophil counts [48,49]. These types of leukocytes are responsible to be in the frontline to fight periodontal infection [50]. If this local infection persists, the bone marrow is signalized to produce a higher number of inflammatory cells [51]. If the periodontal epithelium turns ulcerated, periodontal pathogens may invade the organism and trigger a systemic response to neutralize any harmful consequences [22]. Additionally, clinical situations in which proinflammatory levels are increased (CRP, TNF- $\alpha$ or interleukins) incite the host immune to react against periodontal infection exacerbating the periodontal destruction [52]. Therefore, mediators of inflammation play a key role in the progression of PD, and our results support it regarding DII via the average values of CAL.

Interestingly, PESA accurately measures the surface area of periodontal pocket epithelium [33], but had no significance in this study. Notwithstanding, these results are not surprising since mean PPD is a key measure in PESA computation, though PESA reflects the tridimensional perspective of the periodontium. A limitation is that PESA does not express the surface inflamed area, the so-called periodontal inflamed surface area, as this depends on the existence of bleeding on probing measures, not assessed in the NHANES 2009-2014. Therefore, it will be interesting to study in the future the involvement of the area of periodontal inflammation in this relationship with an inflammatory diet.

In this way, we might hypothesize that PD patients consuming a highly inflammatory diet can not only aggravate clinical symptoms but also be associated with an increased risk towards other relevant systemic conditions, such cardiovascular disease and diabetes where PD plays a key role as a modifying factor $[53,54]$. Both PD and DII present direct links to systemic conditions, but indirectly they might convey to worse systemic status.

We also judged from the analysis of our results that patients who have PD when subjected to an anti-inflammatory diet might demonstrate positive results in involution in the disease, though this warrants confirmation through randomized clinical trials in PD patients.

This investigation presents some limitations. The cross-sectional survey nature does not allow any inference of causality or temporal relationship between the PD and DII. A further limitation is the fact that our analyses were based on dietary self-reported questionnaires, and thereby there is an inherent accepted bias. Also, the DII was computed from 26 food parameters (out of possible 45) from the NHANES questionnaire, yet a previous study has performed this alike [19,55]. Also, the questionnaire refers to a 24 -hour period prior to the interview and this may be seen as a shortcoming [16].

Another important point is the shortage information on systemic inflammation markers, as the CRP levels were only available on NHANES 2009-2010, which limits the validity of these results. Also, data on TNF-a and interleukins would be of interest to study in future research. When exploring the association and mediated effect, these results should be interpreted cautiously, as the causal association will only be possible to prove through prospective studies and randomized intervention trials.

Nevertheless, a number of strengths are worth mentioning. This large sample is based on three consecutive NHANES waves, representative of the American population. The number of covariates included in our analyses were comprehensive and provided consistency to the results. The periodontal assessment was made by calibrated examiners and on a full-mouth basis, increasing the accuracy and precision of the results $[34,56]$.

\section{Conclusions}

inflammatory diet and PD may be associated, as patients with a more pro-inflammatory dietary pattern are more likely to present worse periodontal measures. Also, the inflammatory diet significantly mediated the association of leukocyte counts and systemic inflammation with PD.

Supplementary Materials:, Appendix S1: STROBE-Nut. 
Author Contributions: Conceptualization, V.M., J.B.; methodology V.M., J.B., J.V.; validation, V.M., J.B., L.P., L.L.; formal analysis, J.B., V.M., L.P.; investigation, J.B., V.M.; resources, J.J.M, V.M., J.B., A.S.D.; data curation, J.B., V.M., J.V.; writing - original draft preparation, V.M., J.B., J.V., P.P., L.L., A.S.D., J.J.M.; writing - review and editing, J.B., V.M. All authors have read and agreed to the published version of the manuscript.

Funding: This work is financed by national funds through the FCT - Foundation for Science and Technology, I.P., under the project UIDB/04585/2020.

Acknowledgments: We acknowledge the NHANES investigators and personnel.

Conflicts of Interest: The authors declare no conflict of interest.

\section{References}

1. Bates, C.J.; Hamer, M.; Mishra, G.D. Redox-modulatory vitamins and minerals that prospectively predict mortality in older British people: The National Diet and Nutrition Survey of people aged 65 years and over. Br. J. Nutr. 2011, 105, 123-132.

2. Jia, X.; Aucott, L.S.; McNeill, G. Nutritional status and subsequent all-cause mortality in men and women aged 75 years or over living in the community. Br. J. Nutr. 2007, 98, 593-599.

3. Walston, J.; Xue, Q.; Semba, R.D.; Ferrucci, L.; Cappola, A.R.; Ricks, M.; Guralnik, J.; Fried, L.P. Serum antioxidants, inflammation, and total mortality in older women. Am. J. Epidemiol. 2006, 163, 18-26.

4. Martín-Calvo, N.; Martínez-Gonzàlez, M.Á. Vitamin C intake is inversely associated with cardiovascular mortality in a cohort of spanish graduates: The SUN project. Nutrients 2017, 9, 1-13.

5. Garcia-Arellano, A.; Ramallal, R.; Ruiz-Canela, M.; Salas-Salvadó, J.; Corella, D.; Shivappa, N.; Schröder, H.; Hébert, J.R.; Ros, E.; Gómez-Garcia, E.; et al. Dietary inflammatory index and incidence of cardiovascular disease in the PREDIMED study. Nutrients 2015, 7, 4124-4138.

6. Benjamin, E.J.; Virani, S.S.; Callaway, C.W.; Chamberlain, A.M.; Chang, A.R.; Cheng, S.; Chiuve, S.E.; Cushman, M.; Delling, F.N.; Deo, R.; et al. Heart disease and stroke statistics - 2018 update: A report from the American Heart Association; 2018; Vol. 137; ISBN 0000000000000.

7. Wang, K.; Sun, J.Z.; Wu, Q.X.; Li, Z.Y.; Li, D.X.; Xiong, Y.F.; Zhong, G.C.; Shi, Y.; Li, Q.; Zheng, J.; et al. Long-term anti-inflammatory diet in relation to improved breast cancer prognosis: a prospective cohort study. npj Breast Cancer 2020, 6 .

8. Vadell, A.K.E.; Bärebring, L.; Hulander, E.; Gjertsson, I.; Lindqvist, H.M.; Winkvist, A. Antiinflammatory Diet In Rheumatoid Arthritis ( ADIRA ) - a randomized, controlled crossover trial indicating dieffects on disease activity. 2020, 1203-1213.

9. Woelber, J.P.; Gärtner, M.; Breuninger, L.; Anderson, A.; König, D.; Hellwig, E.; Al-Ahmad, A.; Vach, K.; Dötsch, A.; Ratka-Krüger, P.; et al. The influence of an anti-inflammatory diet on gingivitis. A randomized controlled trial. J. Clin. Periodontol. 2019, 46, 481-490.

10. Watanabe, K. Anti-inflammatory diet: Necessity of scientific spotlight and challenges. Complement. Ther. Med. 2020, 50, 2019-2020.

11. Krebs-Smith, S.M.; Subar, A.F.; Reedy, J. Examining dietary patterns in relation to chronic disease: Matching measures and methods to questions of interest. Circulation 2015, 132, 790-793.

12. Shivappa, N.; Steck, S.E.; Hurley, T.G.; Hussey, J.R.; Hébert, J.R. Designing and developing a literaturederived, population-based dietary inflammatory index. Public Health Nutr. 2014, 17, 1689-1696.

13. Cavicchia, P.P.; Steck, S.E.; Hurley, T.G.; Hussey, J.R.; Ma, Y.; Ockene, I.S.; Hébert, J.R. A New Dietary Inflammatory Index Predicts Interval Changes in Serum High-Sensitivity. J Nutr 2009, 139, 2365-2372.

14. Carson, S.J.; Burns, J. Impact of smoking on tooth loss in adults. Evid. Based. Dent. 2016, 17, 73-74.

15. Niegawa, T.; Kimitaka Takitani, R.T.; Manabu Ishiro, Yuichi Kuroyanagi, Keisuke Okasora, Y.M.; 
Matsuda, T.; Tamai, and H.; Department Evaluation of uric acid levels, thyroid function, the Society for Free Radical Research Japan 1880 50860912-0009 10.3164/j5cb .17-55 JJCBN Kyj bn17-5 Original Article c oto, Japan ournal of Clinical Biochemistry and Nutrition and anthropometric parameters. J. Clin. Biochem. Nutr. 2018, 62, 179-186.

16. Shivappa, N.; Hebert, J.R.; Marcos, A.; Diaz, L.E.; Gomez, S.; Nova, E.; Michels, N.; Arouca, A.; GonzálezGil, E.; Frederic, G.; et al. Association between dietary inflammatory index and inflammatory markers in the HELENA study. Mol. Nutr. Food Res. 2017, 61, 1-23.

17. Ramallal, R.; Toledo, E.; Martínez, J.A.; Shivappa, N.; Hébert, J.R.; Martínez-González, M.A.; RuizCanela, M. Inflammatory potential of diet, weight gain, and incidence of overweight/obesity: The SUN cohort. Obesity 2017, 25, 997-1005.

18. Neufcourt, L.; Assmann, K.E.; Fezeu, L.K.; Touvier, M.; Graffouillère, L.; Shivappa, N.; Hébert, J.R.; Wirth, M.D.; Hercberg, S.; Galan, P.; et al. Prospective association between the dietary inflammatory index and metabolic syndrome: Findings from the SU.VI.MAX study. Nutr. Metab. Cardiovasc. Dis. 2015, 25, 988-996.

19. Kotsakis, G.A.; Chrepa, V.; Shivappa, N.; Wirth, M.; Hébert, J.; Koyanagi, A.; Tyrovolas, S. Diet-borne systemic inflammation is associated with prevalent tooth loss. Clin. Nutr. 2018, 37, 1306-1312.

20. Tonetti, M.S.; Jepsen, S.; Jin, L.; Otomo-Corgel, J. Impact of the global burden of periodontal diseases on health, nutrition and wellbeing of mankind: A call for global action. J. Clin. Periodontol. 2017, 44, 456462.

21. Kassebaum, N.J.; Bernabé, E.; Dahiya, M.; Bhandari, B.; Murray, C.J.L.; Marcenes, W. Global burden of severe periodontitis in 1990-2010: A systematic review and meta-regression. J. Dent. Res. 2014, 93, 10451053.

22. Hajishengallis, G. Periodontitis: From microbial immune subversion to systemic inflammation. Nat. Rev. Immunol. 2015, 15, 30-44.

23. de Pablo, P.; Chapple, I.L.C.; Buckley, C.D.; Dietrich, T. Periodontitis in systemic rheumatic diseases. Nat. Rev. Rheumatol. 2009, 5, 218-224.

24. Buset, S.L.; Walter, C.; Friedmann, A.; Weiger, R.; Borgnakke, W.S.; Zitzmann, N.U. Are periodontal diseases really silent? A systematic review of their effect on quality of life. J. Clin. Periodontol. 2016, 43, 333-344.

25. Botelho, J.; Machado, V.; Proença, L.; Bellini, D.H.; Chambrone, L.; Alcoforado, G.; Mendes, J.J. The impact of nonsurgical periodontal treatment on oral health-related quality of life: a systematic review and meta-analysis. Clin. Oral Investig. 2020, 24, 585-596.

26. Kondo, K.; Ishikado, A.; Morino, K.; Nishio, Y.; Ugi, S.; Kajiwara, S.; Kurihara, M.; Iwakawa, H.; Nakao, K.; Uesaki, S.; et al. A high-fiber, low-fat diet improves periodontal disease markers in high-risk subjects: A pilot study. Nutr. Res. 2014, 34, 491-498.

27. Nascimento, G.G.; Peres, M.A.; Mittinty, M.N.; Peres, K.G.; Do, L.G.; Horta, B.L.; Gigante, D.P.; Corrêa, M.B.; Demarco, F.F. Diet-induced overweight and obesity and periodontitis risk: An application of the parametric g-formula in the 1982 pelotas birth cohort. Am. J. Epidemiol. 2017, 185, 442-451.

28. Raindi, D. Nutrition and periodontal disease. Dent. Update 2016, 43, 66-72.

29. Alhassani, A.A.; Hu, F.B.; Rimm, E.B.; Li, Y.; Rosner, B.A.; Willett, W.C.; Joshipura, K.J. Dietary flavonoid intake and risk of periodontitis. J. Periodontol. 2020, 91, 1057-1066.

30. Lachat, C.; Hawwash, D.; Ocké, M.C.; Berg, C.; Forsum, E.; Hörnell, A.; Larsson, C.; Sonestedt, E.; Wirfält, E.; Åkesson, A.; et al. Strengthening the Reporting of Observational Studies in Epidemiology- 
Nutritional Epidemiology (STROBE-nut): An Extension of the STROBE Statement. PLOS Med. 2016, 13, e1002036.

31. Eke, P.I.; Thornton-Evans, G.O.; Wei, L.; Borgnakke, W.S.; Dye, B.A. Accuracy of NHANES periodontal examination protocols. J. Dent. Res. 2010, 89, 1208-1213.

32. Eke, P.I.; Page, R.C.; Wei, L.; Thornton-Evans, G.; Genco, R.J. Update of the Case Definitions for Population-Based Surveillance of Periodontitis. J. Periodontol. 2012, 83, 1449-1454.

33. Nesse, W.; Abbas, F.; Van Der Ploeg, I.; Spijkervet, F.K.L.; Dijkstra, P.U.; Vissink, A. Periodontal inflamed surface area: Quantifying inflammatory burden. J. Clin. Periodontol. 2008, 35, 668-673.

34. Botelho, J.; Machado, V.; Proença, L.; Mendes, J.J. The 2018 periodontitis case definition improves accuracy performance of full-mouth partial diagnostic protocols. Sci. Rep. 2020, In press.

35. Kaye, E.K. Nutrition, dietary guidelines and optimal periodontal health. Periodontol. 2000 2012, 58, 93111.

36. Laiola, M.; De Filippis, F.; Vitaglione, P.; Ercolini, D. A mediterranean diet intervention reduces the levels of salivary periodontopathogenic bacteria in overweight and obese subjects. Appl. Environ. Microbiol. 2020, 86.

37. Pulido-Moran, M.; Bullon, P.; Morillo, J.M.; Battino, M.; Quiles, J.L.; Ramirez-Tortosa, Mc.C. The relationship between insulin resistance and periodontitis is not affected by Mediterranean diet in a Spanish population. Arch. Oral Biol. 2017, 77, 62-67.

38. Slawik, S.; Staufenbiel, I.; Schilke, R.; Nicksch, S.; Weinspach, K.; Stiesch, M.; Eberhard, J. Probiotics affect the clinical inflammatory parameters of experimental gingivitis in humans. Eur. J. Clin. Nutr. 2011, 65, 857-863.

39. G, G. Probiotics and periodontal health. J. Med. Life 2011.

40. A, C.Ab.Hk. Probiotics in periodontal health and disease. J. Indian Soc. Periodontol. 2011.

41. Hansen, T.H.; Kern, T.; Bak, E.G.; Kashani, A.; Allin, K.H.; Nielsen, T.; Hansen, T.; Pedersen, O. Impact of a vegan diet on the human salivary microbiota. Sci. Rep. 2018, 8, 1-11.

42. Tomofuji, T.; Ekuni, D.; Irie, K.; Azuma, T.; Endo, Y.; Tamaki, N.; Sanbe, T.; Murakami, J.; Yamamoto, T.; Morita, M. Preventive Effects of a Cocoa-Enriched Diet on Gingival Oxidative Stress in Experimental Periodontitis. J. Periodontol. 2009, 80, 1799-1808.

43. Baumgartner, S.; Imfeld, T.; Schicht, O.; Rath, C.; Persson, R.E.; Persson, G.R. The Impact of the Stone Age Diet on Gingival Conditions in the Absence of Oral Hygiene. J. Periodontol. 2009, 80, 759-768.

44. Woelber, J.P.; Bremer, K.; Vach, K.; König, D.; Hellwig, E.; Ratka-Krüger, P.; Al-Ahmad, A.; Tennert, C. An oral health optimized diet can reduce gingival and periodontal inflammation in humans - a randomized controlled pilot study. BMC Oral Health 2017, 17, 28.

45. Giugliano, D.; Ceriello, A.; Esposito, K. The Effects of Diet on Inflammation Emphasis on the Metabolic Syndrome. 2006, 48 .

46. Sproston, N.R.; Ashworth, J.J. Role of C-Reactive Protein at Sites of Inflammation and Infection. Front. Immunol. 2018, 9.

47. Rhodes, B.; Fürnrohr, B.G.; Vyse, T.J. C-reactive protein in rheumatology: Biology and genetics. Nat. Rev. Rheumatol. 2011, 7, 282-289.

48. Craig, R.G.; Yip, J.K.; So, M.K.; Boylan, R.J.; Socransky, S.S.; Haffajee, A.D. Relationship of Destructive Periodontal Disease to the Acute-Phase Response. J. Periodontol. 2003, 74, 1007-1016.

49. Hirschfeld, J. Dynamic interactions of neutrophils and biofilms. J. Oral Microbiol. 2014, 6, 1-10.

50. Hajishengallis, G. New developments in neutrophil biology and periodontitis. Periodontol. 2000 2020, 82, 
78-92.

51. Belkaid, Y.; Hand, T.W. Role of the microbiota in immunity and inflammation. Cell 2014, 157, 121-141.

52. Ebersole, J.L.; Dawson, D.R.; Morford, L.A.; Peyyala, R.; Miller, C.S.; Gonzaléz, O.A. Periodontal disease immunology: "Double indemnity" in protecting the host. Periodontol. 2000 2013, 62, 163-202.

53. Sanz, M.; Marco del Castillo, A.; Jepsen, S.; Gonzalez-Juanatey, J.R.; D’ Aiuto, F.; Bouchard, P.; Chapple, I.; Dietrich, T.; Gotsman, I.; Graziani, F.; et al. Periodontitis and cardiovascular diseases: Consensus report. J. Clin. Periodontol. 2020, 47, 268-288.

54. D' Aiuto, F.; Gkranias, N.; Bhowruth, D.; Khan, T.; Orlandi, M.; Suvan, J.; Masi, S.; Tsakos, G.; Hurel, S.; Hingorani, A.D.; et al. Systemic effects of periodontitis treatment in patients with type 2 diabetes: a 12 month, single-centre, investigator-masked, randomised trial. Lancet Diabetes Endocrinol. 2018, 6, 954-965.

55. Deng, F.E.; Shivappa, N.; Tang, Y.; Mann, J.R.; Hebert, J.R. Association between diet-related inflammation, all-cause, all-cancer, and cardiovascular disease mortality, with special focus on prediabetics: findings from NHANES III. Eur. J. Nutr. 2017, 56, 1085-1093.

56. Machado, V.; Botelho, J.; Mascarenhas, P.; Cavacas, M.A.; Alves, R.; Mendes, J.J. Partial recording protocols performance on the assessment of periodontitis severity and extent: Bias magnitudes, sensibility, and specificity. Rev. Port. Estomatol. Med. Dent. e Cir. Maxilofac. 2018, 59, 145-153. 